Artículo científico

Volumen 32(2):365-381. Mayo-agosto, 2021

e-ISSN 2215-3608, doi:10.15517/am.v32i2.41630

https://www.revistas.ucr.ac.cr/index.php/agromeso

\title{
Composición arbórea de especies asociadas al cacao: selva Lacandona y sistemas agroforestales, Chiapas, México ${ }^{1}$
}

\section{Tree composition of cocoa associated species: Lacandon jungle and agroforestry systems, Chiapas, Mexico}

\author{
Carlos Hugo Avendaño-Arrazate², Gicli Manuel Suárez-Venero , Alexander Mendoza-López², Misael Martínez- \\ Bolaños $^{2}$, Jorge Reyes-Reyes ${ }^{4}$, Saúl Espinosa-Zaragoza
}

1 Recepción: 27 de agosto, 2020. Aceptación: 14 de enero, 2021. Este trabajo formó parte del proyecto de investigación No. 2017-02-291417 y fue financiado por el Fondo SADER-CONACYT-México.

2 Instituto Nacional de Investigaciones Forestales Agrícolas y Pecuarias. Campo Experimental Rosario Izapa, Km. 18 Carretera TapachulaCacahoatán, Tuxtla Chico, CP. 30870, Chiapas, México. Tel. (+52) 962121 0383. avendano.carlos@inifap.gob.mx (autor para la correspondencia; https://orcid.org/0000-0003-3591-2280); martinez.misael@inifap.gob.mx (https://orcid.org/000-0002-8711-8903); amenlo@hotmail. com (https://orcid.org/0000-0003-2146-7163).

3 Universidad de Guantánamo. Avenida Che Guevara, km 1.5 Carretera a Jamaica, Guantánamo, CP. 95 100, Cuba. gicli@cug.co.cu (https:// orcid.org/0000-0002-5235-9192).

4 Universidad Autónoma de Chiapas. Facultad de Ciencias Agrícolas. Carretera Huehuetan Pueblo. Huehuetan, Chiapas. Jorge.reyes@unach. mx (https://orcid.org/0000-0002-5474-2312); saulez1@gmail.com (https://orcid.org/0000-00017683-7382).

\section{Resumen}

Introducción. La composición arbórea asociada al cacao criollo y la que se desarrolla en los sistemas productivos de cacao, es diferente. Esta información es importante para adoptar futuras estrategias en programas de conservación y mejoramiento. Objetivo. Contribuir al conocimiento sobre la composición arbórea de especies asociadas al cacao criollo en México. Materiales y métodos. Los datos analizados en este estudio incluyeron variables de la estructura horizontal y vertical, además de la diversidad de especies arbóreas asociadas al cacao en diferentes condiciones. Este estudio se realizó durante mayo a septiembre del 2018, en parcelas donde se encontraron árboles de cacao criollo en su hábitat natural de la selva Lacandona y en plantaciones de cacao en tres localidades del Soconusco, en Chiapas, México. En las mismas se contabilizaron y clasificaron taxonómicamente las especies arbóreas. Resultados. Asociadas al cacao criollo en la selva Lacandona se registraron veintiún especies, que se agruparon en diecinueve géneros y trece familias, cuya abundancia, riqueza y densidad fue mayor que la registrada en sistemas productivos de cacao en la región del Soconusco, lo que contribuyó a una diferencia entre ambos ecosistemas con solo dos especies compartidas. Conclusión. La abundancia, riqueza y densidad de especies arbóreas asociadas al cacao criollo fue mayor que la registrada en los sistemas de producción, con disimilitud en sus condiciones. Se registraron diferencias en la localización del cultivo del cacao dentro de la estructura vertical de ambos escenarios estudiados.

Palabras clave: Theobroma cacao, estructura vertical, Soconusco, biodiversidad, perennifolia. 


\begin{abstract}
Introduction. The tree composition associated with creole cocoa and that developed in cocoa production systems is different. This information is important to adopt future strategies in conservation and improvement programs. Objective. Contribute to the knowledge about the tree composition of species associated with creole cacao in Mexico. Materials and methods. The data analyzed in this study included variables of the horizontal and vertical structure, in addition to the diversity of tree species associated with cocoa under different conditions. This study was carried out during May to September 2018, in plots where creole cacao trees were found in their natural habitat in the Lacandona jungle and in cacao plantations in three locations in Soconusco, in Chiapas, Mexico. In them, the tree species were counted and taxonomically classified. Results. Associated with the creole cacao in the Lacandon jungle, twenty-one species were registered, which were grouped into nineteen genera and thirteen families, whose abundance, richness and density was greater than that registered in cocoa production systems in the Soconusco region, which contributed to a difference between both ecosystems with only two shared species. Conclusion. The abundance, richness, and density of tree species associated with creole cocoa was higher than that registered in the production systems, with dissimilarity in their conditions. Differences were recorded in the location of the cocoa crop within the vertical structure of both scenarios studied.
\end{abstract}

Keywords: Theobroma cacao, vertical structure, Soconusco, biodiversity, evergreen.

\title{
Introducción
}

México es uno de los países productores de cacao en América cuyos rendimientos aún no sobrepasa el 50 $\%$ de la producción en los principales países productores el mundo. Sus plantaciones se encuentran concentradas mayormente en los estados de Tabasco y Chiapas. Particularmente en Chiapas, las principales regiones de producción son el Norte, Centro, Soconusco y Selva-Norte, con rendimientos promedios en los últimos años que varían entre 0,20 - 0,54 t ha ${ }^{-1}$ (Servicio de Información Agroalimentaria y Pesquera, 2018). Dentro de este estado se encuentra la selva Lacandona o también llamada Desierto de la Soledad, una de las zonas de mayor biodiversidad del país y del trópico húmedo de América.

Dentro de los estudios realizados en el área geográfica de la selva Lacandona, son escasos los comentarios que se han hecho sobre el registro del cacao criollo en su hábitat natural; este es un recurso genético que se ha conservado durante muchos años en los ambientes totalmente aislados de poblaciones comerciales (AvendañoArrazate et al., 2018). Sin embargo, esta especie se encuentra desarrollada en su estado natural y de forma silvestre en convivencia con diferentes especies típicas de la selva alta perennifolia. En este contexto, los estudios de su composición arbórea en condiciones naturales aún son escasos. Lo mismos permitirían emitir criterios sobre su hábitat natural y diversidad, que pueden ser comparados con otros agroecosistemas, como los agroforestales, dentro de los que se encuentran los actuales sistemas productivos de cacao.

El conocimiento de la composición arbórea en los sistemas productivos de cacao, permitirán reconocer el uso y aprovechamiento de la diversidad dentro del propio agroecosistema a partir del valor de importancia de cada una de las especies forestales que se relacionan con el cultivo y, así poder diseñar sistemas agroforestales más eficientes. Este aprovechamiento puede ser observado desde tres impactos fundamentales: sobre el propio cultivo, sobre la dinámica medioambiental y sobre la vida socioeconómica de los productores.

Las especies forestales con funciones para sombra en las plantaciones de cacao, permiten el normal crecimiento y desarrollo fisiológico del cultivo, al contrabalancear aquellos factores que son perjudiciales cuando 
el cultivo se encuentra expuesto a una mayor radiación. Una composición sombreadora adecuada en plantaciones en producción, podría dejar pasar de un 60-80 \% de la iluminación total (Somarriba, 2004) y con ello la energía suficiente para el desarrollo del proceso fotosintético, la activación de yemas reproductivas, la apertura estomática, y la expansión celular de ciertos tejidos (Alvim, 1977).

Desde el punto de vista medioambiental, una diversidad apropiada de dicha composición podría influir positivamente sobre el suelo, evitando el impacto directo de las gotas de agua, manteniendo la humedad y aportando nutrientes por medio de la biomasa de dichas especies forestales, en beneficio de la propia plantación. También, una de las formas para mitigar el cambio climático es la reducción de las concentraciones de $\mathrm{CO}_{2}$ en la atmósfera, a través del establecimiento de sistemas agroforestales (SAF), capaces de capturar el $\mathrm{CO}_{2}$ y almacenarlo en la biomasa aérea y subterránea por largos periodos (Gayoso \& Guerra, 2005). Los aspectos mencionados podrían lograr que los sistemas productivos de cacao sean más eficientes, ello con el conocimiento de la composición sombreadora.

La diversidad de especies para sombra en los agroecosistemas cacaoteros, colabora con la calidad de vida de los productores con base en el uso e importancia de cada una de las especies (madera, fibras, frutas, leña, medicinas, etc.) y mayores ingresos familiares, por ello la importancia de profundizar en el conocimiento de su composición. Si esta diversidad es manejada adecuadamente, se pueden lograr importantes beneficios para el cultivo (Silva et al., 2013). Los sistemas agroforestales (SAF) con cacao son valiosos para la conservación de la Biodiversidad, ya que su alta diversidad vegetal provee de hábitats, nichos y alimentos a otras especies de plantas y animales, amortiguan las áreas protegidas y sirven de conexión entre los ecosistemas intactos y manejados del paisaje. Sin embargo, dicha diversidad debe ser correctamente manejada en función de beneficiar los procesos fisiológicos del cultivo (Rice \& Greenberg, 2000).

Como antecedentes de estas investigaciones relacionado con el cultivo del cacao, en México se han desarrollado algunos estudios por Ramírez-Meneses et al. (2013) en el estado Tabasco; Roa-Romero et al. (2009) y SalgadoMora et al. (2007) en el Soconusco, Chiapas.

Otros estudios florísticos, pero en escenarios típicos de la selva, se han realizado en la Amazonía (Burnham 2004; de-Oliveira \& Nelson, 2001; Nebel et al., 2001a; b; Pitman et al., 2002; Parolin et al., 2004; Poulsen et al., 2006; Wittmann et al., 2006), por lo que se tiene resultados de la composición florística y la diversidad vegetal de esta región, fundamentalmente pertenecientes a Ecuador, Perú y Brasil, sin tener en cuenta como objeto de estudio el cultivo del cacao.

En el sudeste del estado Bahía en Brasil, la CEPLAC (Comissão Executiva do Plano da Lavoura Cacaueira) ha ejecutado diferentes estudios, entre ellos lo relacionado con la identificación de la preferencia de los productores de árboles locales de sombra en el sistema cacao-cabrucas donde se da prioridad a los árboles nativos para conservar la diversidad biológica y el aprovechamiento de sus usos (Bonazzi et al., 2014).

Cálculos conservadores afirman que la riqueza de especies en la selva Lacandona se aproxima a los 500 árboles ha $^{-1}$ en tan solo $0,16 \%$ de la superficie del país. Se considera que esta región cuenta con más de $20 \%$ del total de las especies existentes en México (Durán-Fernández et al., 2016).

En la región que ocupa la selva Lacandona, los antecedentes bibliográficos sobre el tema, muestran un listado informativo sobre la vegetación original y secundaria, e incluyen listas de especies presentes en sus áreas de estudio respectivas sin mencionar el cultivo del cacao, entre ellos Durán-Fernández et al. (2016), Escobar-Campos \& Ochoa-Gaona (2007), Levy-Tacher et al. (2002; 2006) y Ochoa-Gaona et al. (2007), aunque estos últimos autores refieren al Theobroma bicolor L. Sin embargo, dentro de los escasos estudios relacionados con Theobroma cacao L. en la región, se encuentra el realizado por López-Baez et al. (2015), quienes evaluaron la diversidad y composición de las plantaciones de cacao en la Reserva de la Biosfera Montes Azules, perteneciente al municipio Maravilla Tenejapa y se ubica en la región económica XII Selva Lacandona.

El objetivo de la presente investigación fue contribuir al conocimiento sobre la composición arbórea de especies asociadas al cacao criollo en México. 


\section{Materiales y Métodos}

La investigación se desarrolló entre mayo y septiembre del 2018 en el sitio denominado El Paso del Tapir de la selva Lacandona, ubicada a una altitud de $326 \mathrm{~m}$, sobre terreno semi ondulado, pendiente del $10 \%$, orientación de la ladera norte y sin actividad antropogénica, y en algunas localidades de tres municipios donde se desarrolla los sistemas productivos de cacao en el Soconusco, ambas regiones del estado Chiapas, México.

La selva Lacandona se localiza al oriente del estado de Chiapas, extendiéndose hasta el extremo noroccidental del Estado. La misma se encuentra ubicada entre los $16^{\circ} 17^{\prime}$ de latitud norte y los $90^{\circ} 30^{\prime}$ de longitud oeste del estado.

Según el sistema de Köppen modificado por García (1973), el clima de esta región que ocupa la selva Lacandona, es predominantemente cálido húmedo, con una distribución y temperatura relativamente homogéneas, cuyos valores de temperatura media anual se encuentran entre 18 y $22{ }^{\circ} \mathrm{C}$ y baja oscilación térmica anual. Las lluvias alcanzan valores anuales superiores a los $1500 \mathrm{~mm}$ y pueden llegar hasta los $3000 \mathrm{~mm}$ en la zona norte. Las temperaturas, la humedad y la lluvia presente en la mayor parte del año presente favorecen los procesos de alteración de minerales del suelo, así como la degradación de la abundante materia orgánica acumulada (DuránFernández et al., 2016).

El área que ocupa la región del Soconusco se encuentra ubicada al sur del estado de Chiapas, México y está localizada entre las coordenadas $15^{\circ} 19^{\prime} \mathrm{N}$ y $92^{\circ} 44^{\prime} \mathrm{W}$, cubriendo $4605,4 \mathrm{~km}^{2}$, que representan el $6,28 \%$ de la superficie estatal; es la séptima región de mayor extensión territorial en el estado de Chiapas.

Los climas que se presentan en la región del Soconusco son los cálidos y semicálidos. Predomina el clima cálido subhúmedo con lluvias de verano, seguido por el cálido húmedo con lluvias abundantes de verano. Por tanto, de acuerdo a la clasificación de Köppen modificado por García (1973), el clima predominante en la región de estudio es del tipo Aw2 (w) Ig con humedad relativa media de $79,4 \%$ y la temperatura media anual de $26,8{ }^{\circ} \mathrm{C}$ (Comisión Nacional del Agua, 2015).

\section{Muestreo}

Para realizar el muestreo e inventario de las especies de sombra asociadas a los árboles de cacao (Theobroma cacao L.) en ambas regiones de estudio, se partió del criterio de homogeneidad de superficie muestreada para ambas condiciones y se consideró el número limitado de árboles de cacao existentes en el sector estudiado de la selva Lacandona. Por lo tanto, estas últimas condiciones definieron la cantidad de parcela muestreada en cada condición.

Con la colaboración del personal técnico que laboran en la región estudiada de la selva, se localizaron los árboles de cacao criollo existente que se desarrollan en sus condiciones naturales. Luego de varios recorridos y observaciones, se detectaron solo tres árboles de cacao, los cuales se encontraban ubicados a una distancia promedio de $500 \mathrm{~m}$. Alrededor de los mismos se establecieron las parcelas de forma tal que los árboles de cacaos criollos quedaran en el centro de las mismas. El muestreo se realizó en tres parcelas rectangulares con una dimensión de $20 \mathrm{~m}$ x $50 \mathrm{~m}(0,1 \mathrm{ha})$ que se correspondieron con los tres árboles de cacao existentes, para un total de superficie muestreada en la selva de 0,3 ha.

Para cumplir con el principio de homogeneidad de superficie explicado con anterioridad, en el Soconusco el muestreo se realizó en tres parcelas pertenecientes a tres localidades que correspondieron a tres municipios donde se desarrollan los sistemas productivos de cacao (Cuadro 1). De esta manera, se realizó un muestreo dentro de las plantaciones de cacao con parcelas rectangulares con las mismas características que las utilizadas en la selva, para un área total de 0,3 ha.

Para determinar las localidades y zonas de muestreo en estos sistemas productivos, se realizó previamente un recorrido exploratorio con los actores directos de la producción en el $90 \%$ de las plantaciones de cacao en cada municipio. A partir de este recorrido, se logró definir los indicadores con sus criterios para seleccionar los municipios 
Cuadro 1. Localidades evaluadas con sistemas productivos de cacao (Theobroma cacao L.) en la región Soconusco, Chiapas, México, 2018.

Table 1. Localities evaluated with productive systems of cocoa (Theobroma cacao L.) in the Soconusco region, Chiapas, Mexico, 2018.

\begin{tabular}{cccccc}
\hline Municipios & Localidades & Propietarios* & Coord X & Coord Y & Altitud (m) \\
\hline Escuintla & El triunfo & GEM & 544935 & 1697062 & 36 \\
Frontera Hidalgo & La primavera & SGV & 587577 & 1635447 & 83 \\
Acacoyagua & Los cacaos & AMP & 536672 & 1701221 & 398 \\
\hline
\end{tabular}

GEM: Gabriel Escobar Mejía; DGV: Santiago Galindo Vázquez; AMP: Adelita Miranda Paz.

objeto de intervención y las entidades productivas (fincas) a evaluar. Los indicadores definidos estuvieron en función de la representatividad a nivel estatal. Los mismos fueron: 1) superficie plantada con el cultivo; 2) cantidad de productores en la actividad cacaotera 3) porcentaje del aporte a la producción y 4) representatividad geográfica por niveles de altitud desde 0 hasta $500 \mathrm{~m}$. Fueron seleccionados aquellos municipios con los mayores valores de los indicadores estudiados.

En todas las parcelas y en ambas condiciones se registró y cuantificó las especies arbóreas asociadas al cultivo que tuvieron solo un área basal con DAP a 1,3 m sobre el nivel del suelo mayor de $5 \mathrm{~cm}$ medido con una cinta diamétrica de $5 \mathrm{~m}$ (Jackson, MS), además de medir la altura con un clinómetro marca SUUNTO.

Para el caso particular del muestreo realizado en la selva, de cada especie se registró el nombre común según la unión de criterios y experticia de cinco trabajadores que acompañaron la investigación y que poseen más de diez años de experiencias en la realización de inventarios e identificación de especies pertenecientes a una de las siete áreas protegidas de la selva Lacandona que es la Reserva de la Biosfera Montes Azules.

Para todos los casos, se colectaron los órganos (ramas jóvenes, hojas, flores y frutos) por cada especie registrada, los cuales se depositaron en un medio preparado para su herborización, traslado y posterior identificación. El registro de dichos nombres permitió su determinación botánica y clasificación taxonómica (familia, género y especie), apoyados en el manual árboles de Centroamérica del Centro Agronómico Tropical de Investigación y Enseñanza (CATIE) (Cordero \& Boshier, 2003) y árboles tropicales de México (Pennington \& Sarukhán, 2005).

\section{Estructura horizontal}

Se calculó el índice de valor de importancia (IVI) según Curtis \& McIntosh (1951), que define cuáles de las especies presentes contribuyen en el carácter y estructura de un ecosistema. Para ello, se utilizó la fórmula 1, donde, la abundancia relativa es la proporción porcentual de cada especie en el número total de especies; dominancia relativa es la proporción de una especie en el área basal total evaluada y la frecuencia relativa es el porcentaje en la sumatoria de las frecuencias absolutas de todas las especies.

$$
I V I=\text { Abundacia relativa }+ \text { Dominancia relativa }+ \text { Frecuencia relativa }
$$

A partir de las dimensiones de cada parcela de muestreo $(0,1$ ha) y la cuantificación del número de especies y árboles sombreadores, se determinó la densidad de especie y árboles por hectárea. 


\section{Estructura vertical}

Se evaluó la estratificación vertical dada por la distribución de las copas en el perfil vertical respecto a los árboles de cacao nativo en cada una de las parcelas. Se identificaron las especies presentes en los estratos inferior, medio y superior. La misma fue clasificada según Somarriba et al. (2004) en correspondencia con la altura de las especies arbóreas en:

a) Estrato inferior: especies que ocupaban una altura entre 2-8 m.

b) Estrato medio: especies que ocupaban una altura entre 9-25 m.

c) Estrato superior: especies que ocupaban una altura entre 26-40 m.

\section{Análisis de similitud con los sistemas de producción}

Se realizó un análisis comparativo de similitud entre las especies asociadas al cacao de la selva Lacandona, con las especies registradas en los sistemas productivos de cacao en el Soconusco, Chiapas; con el objetivo de evaluar que tan similares fueron las composiciones arbóreas asociadas en ambas condiciones. Para ello, se determinó el coeficiente de Jaccard (Cj) y se utilizó el método de Czekanowski - Sørensen, ambos son métodos cualitativos explicados por Polo-Urrea (2008).

Para determinar el coeficiente de Jaccard $(\mathrm{Cj})$ se utilizó la fórmula 2. Esta se basa en la relación de presenciaausencia entre el número de especies en cada sistema y el número total de especies (Stiling, 1999).

$$
\mathrm{C} j=\frac{\mathrm{C}}{\mathrm{A}+\mathrm{B}-\mathrm{C}}
$$

Donde:

$\mathrm{C}=$ número de especies comunes a ambos sistemas.

$\mathrm{A}=$ número de especies encontradas en el sistema $\mathrm{A}$.

$\mathrm{B}=$ número de especies encontradas en el sistema $\mathrm{B}$.

El método de Czekanowski - Sørensen se basa también en la relación presencia-ausencia entre el número de especies compartidas o no en cada sistema y el número total de especie de los dos sitios en comparación (Fórmula 3).

$$
\frac{2 C}{S 1+S 2}
$$

Donde:

$\mathrm{C}=$ número de especies compartidas entre los dos sitios

$\mathrm{S} 1=$ número de especies en el sitio 1

S2= número de especies en el sitio 2

Para la evaluación de la similitud se utilizó el método cuantitativo de Morisita-Horn según la ecuación de la fórmula 4. Esta considera los valores de las especies compartidas o no entre los dos sitios en comparación y significa la similitud de dos sitios en estructura tanto en composición como en abundancia relativa. Para ello se utilizó la ecuación 4:

$\frac{2 \sum(\text { nia*nib) }}{(\mathrm{da}+\mathrm{db})^{*}\left(\mathrm{Na} \mathrm{N}^{*} \mathrm{Nb}\right)}$


Donde:

nia= número de árboles de la especie $\mathrm{i}$ en el sitio a.

nib= número de árboles de la especie i en el sitio b.

$\mathrm{Na}=$ número de árboles en el sitio a.

$\mathrm{Nb}=$ número de árboles en el sitio b.

Para el sitio a (ecuación 5):

$$
\mathrm{da}=\frac{\sum \mathrm{nia}^{2}}{\mathrm{~N}^{2} \mathrm{a}}
$$

Para el sitio b (ecuación 6):

$$
\mathrm{db}=\frac{\sum \mathrm{nib}^{2}}{\mathrm{~N}^{2} \mathrm{~b}}
$$

\section{Análisis estadístico}

Los datos de la información que se originó del registro de las especies, se sistematizaron y registraron en una base de datos creado con el paquete de Excel versión 9.1, donde se calcularon los índices evaluados y el promedio de especies.

\section{Resultados}

\section{Estructura horizontal}

Asociadas al cacao criollo se registraron un total de veintiún especies, las cuales se agruparon en diecinueve géneros y trece familias. Los géneros más representados fueron: Guarea, Brosimum y Dialium, que en su conjunto concentra un 55,1\% del total de árboles inventariados. Las familias botánicas con mayor número de especies fueron: Magnoliaceae, Meliaceae y Fabaceae, con 23, 22 y 16 árboles, respectivamente. Estas familias agruparon al 62,2 $\%$ del total de árboles inventariados (Cuadro 2). Sin embargo, bajo las condiciones de los sistemas productivos, se registraron dieciséis especies, que se agruparon en catorce géneros y once familias. Los géneros más representados fueron: Cordia, Manguifera y Persea, que concentró el 54,09 \% del total de los árboles registrados. Las familias más representadas fueron: Boraginaceae, Meliaceae y Anacardiaceae, con 25, 8 y 7 árboles, respectivamente. Las mismas agruparon el 65,5\% del total de los árboles inventariados (Cuadro 3).

La riqueza de especies asociadas al cacao criollo en la selva Lacandona (21 especies) fue mayor que la registrada en los sistemas productivos (dieciséis especies). Estas últimas las seleccionaron los productores como sombra de la vegetación del bosque natural y vegetación secundaria por regeneración natural.

En condiciones de la selva, la cantidad de especies y árboles por superficie fue mayor, con una densidad de 70 especies ha-1 y 326 árboles ha-1, mientras que en los sistemas productivos se determinó una densidad de especies y árboles por hectárea de 53,3 y 203, respectivamente.

La abundancia de especies asociadas al cacao criollo en toda el área muestreada fue de 98 árboles. Guarea glabra Vahl, Brosimum alicastrum Swartzy y Dialuim guianense (Aubl.) Sandwith, fueron las más abundantes con 22, 20 y 12 árboles, respectivamente. Estas especies junto a Sapindus saponaria L. resultaron ser las más frecuentes en las parcelas muestreadas. Sin embargo, la abundancia de especies en los sistemas productivos de cacao fue de 
Cuadro 2. Listado de especies arbóreas y su abundancia, asociadas al cacao criollo (Theobroma cacao L.) en la selva Lacandona en Chiapas, México, 2018.

Table 2. List of tree species and their abundance, associated with native cocoa (Theobroma cacao L.) in the Lacandon jungle in Chiapas, Mexico, 2018.

\begin{tabular}{|c|c|c|c|}
\hline Familias & Especies & Nombre común & Abundancia \\
\hline Anacardiaceae & Spondias purpurea $\mathrm{L}$. & ramón ciruela & 5 \\
\hline Anacardiaceae & Spondias mombin L. & Jobo & 1 \\
\hline Annonaceae & Stenanona stenopetala (Donn.Sm.) & anona & 1 \\
\hline Boraginaceae & Cordia alliodora (Ruiz\& Pav.) Oken & laurel & 2 \\
\hline Cecropiaceae & Cecropia obtusifolia Bertol & guarumbo & 2 \\
\hline Combretaceae & Terminalia amazonía (J. F. Gmel.) Exell & canshan & 1 \\
\hline Piperaceae & Piper aduncum L. & cordoncillo & 4 \\
\hline Clethraceae & Clethra occidentalis (L.) O. Kuntze & zapotillo & 4 \\
\hline Fabaceae & Schizolobium parahyba Müll. Arg & zope & 1 \\
\hline Fabaceae & Erythrina berteroana Urb. & colorín & 1 \\
\hline Fabaceae & Erythrina americana Mill. & colorín espinoso & 1 \\
\hline Fabaceae & Dialuim guianense (Aubl.) Sandwith & huapaque & 12 \\
\hline Fabaceae & Cojoba arborea (L.) Britton et Rose & frijolillo & 1 \\
\hline Lauraceae & Ocotea austinii Allen & aguacatillo & 4 \\
\hline Magnoliaceae & Quararibea funebris (La Llave) Vischer & molinillo & 2 \\
\hline Meliaceae & Guarea glabra Vahl & cedrillo & 22 \\
\hline Moraceae & Castilla elastica Sesse & hule silvestre & 1 \\
\hline Moraceae & Brosimum alicastrum $\mathrm{Swartz}$ & ramón rojo & 20 \\
\hline Moraceae & Ficus sp. & matapalo & 2 \\
\hline Sapindaceae & Sapindus saponaria $\mathrm{L}$. & limoncillo & 6 \\
\hline Ulmaceae & Ampelocera hottlei (Stand1.) Standl & cuerillo & 5 \\
\hline
\end{tabular}

Cuadro 3. Listado de especies arbóreas y su abundancia, como sombra asociada al cacao (Theobroma cacao L.) cultivado en los sistemas productivos en el Soconusco, Chiapas, México, 2018.

Table 3. List of tree species and their abundance, as shade associated with cocoa (Theobroma cacao L.) grown in systems of production in Soconusco, Chiapas, Mexico, 2018.

\begin{tabular}{llcc}
\hline Familia & Especie & Nombre común & Abundancia \\
\hline Anacardiaceae & Mangifera indica L. & mango & 7 \\
Bignoniaceae & Tabebuia donnell-smithii Rose & primavera & 4 \\
Bignoniaceae & Tabebuia rosea (Bertol) DC. & roble & 1 \\
Boraginaceae & Cordia alliodora (Ruiz\& Pav.) Oken & laurel & 20 \\
Burseraceae & Bursera simaruba (L.) Sarg. & jiote & 1 \\
Combretaceae & Terminalia amazonia (J.F.Gmel.) Exell & guayabo volador & 2 \\
Fabaceae & Inga micheliana Harms & chalum & 2 \\
Lauraceae & Persea americana Mill. & aguacate & 6 \\
Meliaceae & Cedrela odorata L. & cedro & 4 \\
Meliaceae & Guarea glabra Vahl. & cedrillo & 4 \\
Rutaceae & Citrus nobilis Lour & mandarina & 1 \\
Rutaceae & Citrus sinensis L. & naranja & 1 \\
Sapindaceae & Nephelium lappaceum L. & rambután & 2 \\
Sapotaceae & Manilkara zapota (L.) van Royen & chicozapote & 1 \\
Sapotaceae & Pouteria sapota (Jacq.) H. E. Moore \& Stearn & zapote & 4 \\
Tiliaceae & Apeiba tibourbou Aubl. & peine de mico & 1 \\
\hline
\end{tabular}


61 árboles, siendo Cordia alliodora, Mangifera indica L. y Persea americana Mill las especies más abundantes con 20,7 y 6 , respectivamente.

Con base en la superficie muestreada de forma homogénea en ambas condiciones, tanto en las condiciones de la selva Lacandona como en las plantaciones comerciales de cacao, las especies más abundantes resultaron ser las más dominantes, debido a su diámetro basal y, por consiguiente, las de mayor índice de valor de importancia (Figura 1).
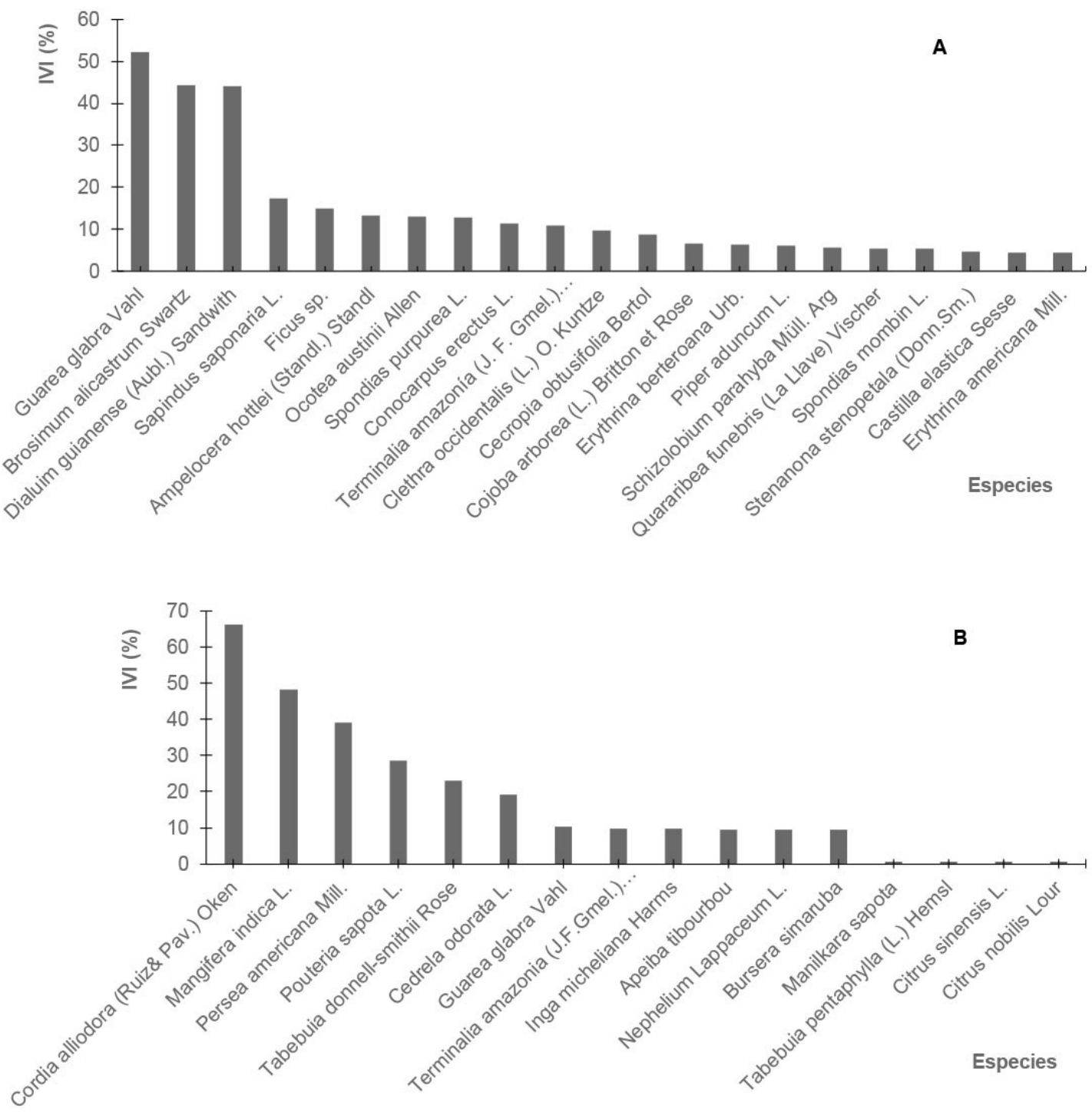

Figura 1. Índice de valor de importancia de las especies arbóreas asociadas al cacao (Theobroma cacao L.) nativo en la selva Lacandona (A) y al cacao desarrollado en los sistemas productivos (B). Chiapas, México, 2018.

Figure 1. Importance value index of tree species associated with native cocoa (Theobroma cacao L.) in the Lacandon jungle (A) and cocoa developed in production systems (B). Chiapas, Mexico, 2018. 
En la selva Lacandona las especies más dominantes fueron: Dialuim guianense, Guarea glabra y Brosimum alicastrum, las cuales ocuparon un área basal de 24,7 $\mathrm{m}^{2} \mathrm{ha}^{-1}, 22,8 \mathrm{~m}^{2} \mathrm{ha}^{-1}$ y $16,3 \mathrm{~m}^{2} \mathrm{ha}^{-1}$, respectivamente. En los sistemas productivos fueron Mangifera indica L., Cordia alliodora (Ruiz \& Pav.) Oken y Persea americana Mill las que ocuparon un área basal de $14,4 \mathrm{~m}^{2} \mathrm{ha}^{-1}, 11,3 \mathrm{~m}^{2} \mathrm{ha}^{-1}$ y $8,7 \mathrm{~m}^{2} \mathrm{ha}^{-1}$, respectivamente.

\section{Estructura vertical}

El 55,4 \% y 42,5 \% de los árboles asociados al cacao criollo ocuparon los estratos medio y superior, respectivamente; mientras que en los sistemas productivos de cacao en el Soconusco las especies registradas ocuparon los estratos inferior y medio (Figura 2).

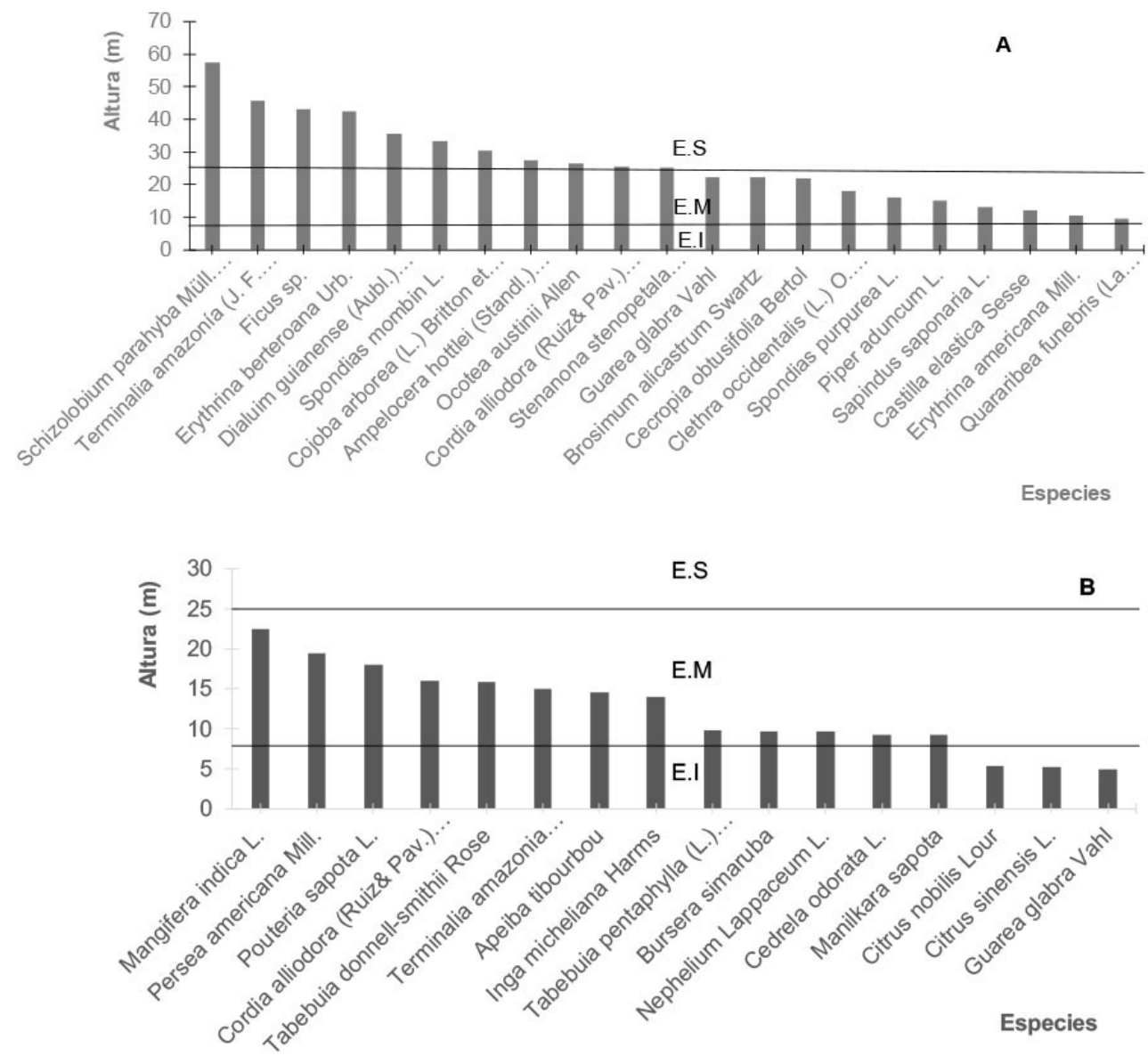

Figura 2. Estructura vertical de las especies arbóreas asociadas al cultivo del cacao (Theobroma cacao L.) nativo en la selva Lacandona (A) y al cacao desarrollado en los sistemas productivos (B). Chiapas, México, 2018.

E.I= estrato inferior, E.M= estrato medio, E.S= estrato superior.

Figure 2. Vertical structure of the species associated with the cultivation of native cocoa (Theobroma cacao L.) in the Lacandona jungle (A) and the cocoa developed in production systems (B). Chiapas, Mexico, 2018.

E.I = lower layer, E.M = middle layer, E.S = upper layer. 
Los árboles de cacao criollo que se desarrollaban en su hábitat natural en la selva Lacandona, se encontraban en el estrato medio, con alturas entre 10,6 y 17,3 m y compartiendo el espacio con diecinueve especies asociadas. Sin embargo, el cacao que se desarrollaba en los sistemas productivos ocupaba alturas promedio entre 4,6 y $8,8 \mathrm{~m}$, debido a las actividades de manejo, lo que hace posible que en estos sistemas dichas plantas se encuentren en el estrato inferior y compartiendo el mismo espacio vertical con un $50 \%$ del total de los árboles registrados.

\section{Análisis de similitud}

A partir de los resultados de los métodos cualitativos y cuantitativos para evaluar la similitud de la composición de especies arbóreas en ambas zonas estudiadas, se determinaron divergencias entre ambos escenarios donde se desarrollaba el cultivo, según los valores obtenidos por el índice de Jaccard y los métodos de Czekanowski Sørensen y Morisita-Horn con 0,057, 0,10 y 0,19, respectivamente. Ello se debió fundamentalmente al registro de solo dos especies compartidas entre ambas condiciones (Cordia alliodora (Ruiz \& Pav.) Oken y Guarea glabra Vahl), por la influencia de condiciones físico-geográfico diferentes y disimilitud en las acciones antropogénicas donde la tecnología de manejo del cultivo en los sistemas de producción en el Soconusco a diferencia de las condiciones selváticas, han jugado un papel fundamental en las constantes transformaciones del escenario productivo.

\section{Discusión}

Con base en el nivel local del estudio en la presente investigación, se logró un inventario del 0,48 \% del número de especies estimado en la región, debido a que esta diversidad no se distribuye de manera uniforme y hay una alta variación de lugar a lugar (Román et al., 2009). La densidad de especies y de árboles de cacao evaluados en las condiciones selváticas es una característica distintiva del hábito, crecimiento y desarrollo de la vegetación en la selva alta perennifolia. Estas selvas tropicales tienen una gran variedad de especies de árboles forestales, por lo que no es raro encontrar más de 200 especies ha ${ }^{-1}$ de bosque tropical. Inventarios realizados en la comunidad Lacandona el Nahá, Chiapas México, registraron 234 árboles ha ${ }^{-1}$ (Durán-Fernández et al., 2016). La selva amazónica y, especialmente, la parte oeste de la cuenca amazónica en Ecuador, Colombia y Perú, tiene los números más altos de especies de plantas viviendo en una hectárea de bosque (Butler, 2011).

En la presente investigación se registró una densidad de árboles ha ${ }^{-1}$ y diversidad muy alta, lo que puede estar relacionado con la falta de las sucesivas regulaciones de sombra que son necesarias para garantizar una adecuada iluminación a lo interior e las plantaciones, según la tecnología para el manejo de los árboles de sombra acorde a los requerimientos del cultivo. Por lo tanto, las condiciones existentes presuponen una limitante para el normal crecimiento, desarrollo y producción del cultivo.

Al respecto del dosel de sombra, se pudo observar que las especies de mayor altura y amplio dosel de sombra aportaban una densa sombra en mayor cantidad de horas. Se ha sugerido que el recorrido sobre el suelo de la sombra de la copa de un árbol a mayor altura, es más largo que el recorrido de la sombra de la copa de un árbol a baja altura (Somarriba, 2002); se ha afirmado que entre los atributos deseables de los árboles de sombra está la altura media entre 15 y 25 m (Silva et al., 2013). Se ha registrado en el estrato superior de los SAF de cacao en Centroamérica, especies que alcanzaron una altura de $36 \mathrm{~m}$, DAP de $137 \mathrm{~cm}$ y área basal media de $11 \mathrm{~m}^{2} \mathrm{ha}^{-1}$ a 47,2 $\mathrm{m}^{2}$ ha $^{-1}$ (Ramírez-Meneses et al., 2013).

Se ha sugerido que la densidad de siembra para las plantaciones en producción de cacao con plantas desarrolladas y autosombreadas, debe ser entre 25 y 50 árboles ha ${ }^{-1}$ para originar 60-75 \% de luz total (González, 2008). Esta información coincide con lo afirmado por Somarriba (2004), quien además destacó que esta iluminación depende del manejo agronómico del cacao y de las características del dosel de sombra. Estudios de SAF en Tabasco, México, 
registraron una densidad media de 286 árboles ha-1 $^{-1}$, variando de 96 a 618 árboles (Sánchez-Gutiérrez et al., 2016). En la misma región del Soconusco otros estudios sobre el tema desarrollados por Suárez-Venero et al. (2018) determinaron una densidad entre 80 a 300 árboles ha $^{-1}$ y en Costa Rica, Somarriba et al. (2000) registraron 278 árboles ha $^{-1}$. La diferencia registrada entre el estudio actual y los mencionados es razonable, ya que la composición arbórea asociada al cacao como sombra en los SAF varía entre los países y entre las regiones dentro de un mismo país.

El conocimiento del valor de importancia calculado en las especies asociadas al cacao criollo, además de su composición arbórea, supone la adopción de medidas de conservación de las mismas sobre la base de preservar su nicho ecológico natural independientemente del uso que poseen las mismas. Este aspecto es positivo para lograr el mantenimiento y la preservación de las especies que resguardan al cacao criollo en sus condiciones naturales. Estas especies sirven de alimento a la propia fauna silvestre de la región como monos saraguato, aves, tepezcuintles, entre otros; que al mismo tiempo sirven de dispersores naturales de fuentes semilleras entre ellas el cacao nativo, lo que contribuye a la dispersión y conservación del mismo en su hábitat natural, como lo hacen los animales frugívoros que contribuyen a la regeneración de las poblaciones de plantas (Schupp et al., 2010).

El sistema de conservación de la región como Reserva de la Biosfera, ha hecho posible la conservación de una buena parte de la selva incluyendo la localidad estudiada con muy poca antropización en la actualidad, a diferencia de otras localidades de esta región donde la Secretaría de Medio Ambiente y Recursos Naturales, ha reconocido que la agricultura extensiva itinerante mediante la roza-tumba-quema con diferentes periodos de barbecho y la ganadería extensiva, han sido las causas de la deforestación en la selva Lacandona (Romaní-Cortes, 2009), ya que según la Comisión Nacional para el Conocimiento y Uso de la Biodiversidad (Comisión Nacional para el Conocimiento y Uso de la Biodiversidad, 2018), las selvas han sido tradicionalmente fuente de maderas preciosas, leña y diversidad de plantas y animales para la subsistencia de comunidades rurales e indígenas.

La composición arbórea registrada en los sistemas productivos de cacao en el Soconusco, es típica de los sistemas agroforestales con cacao de esta región, con especies comunes de la selva alta perennifolia como Terminalia amazonia (J.F.Gmel.) Exell y Cordia alliodora (Ruiz \& Pav.) Oken, criterios que coinciden con las afirmaciones de Roa-Romero et al. (2009). Por lo general, el tipo de sombra presente en las plantaciones estudiadas definió diversos sistemas de producción familiar, donde los productores obtienen productos de autoabastecimiento para el pequeño comercio, leña como energía o para buscar opciones de sustituir el cacao con algunas especies (forestales, frutales, condimentosas) en caso de que decidieran hacer un cambio de uso del suelo (HernándezGómez et al. 2015); tal es el caso de las especies determinadas con mayor valor de importancia asociadas al cacao en las localidades estudiadas del Soconusco.

Entre las especies más importantes se encontraba Cordia alliodora (Ruiz\& Pav.) Oken, cuya popularidad radica en el alto valor de su madera para uso local y puede ser combinada con cultivos anuales y perennes en sistemas agroforestales, además de su uso como planta medicinal en México (Cordero \& Boshier, 2003). Sin embargo, Mangifera indica L. y Persea americana Mill, son especies tradicionales en los SAFc que se han establecido o mantenido de acuerdo a las necesidades de los productores por sus usos en la alimentación, ingresos secundarios de los productores y el aprovechamiento de su biomasa para leña como energía. Estas especies frutales no se registraron en el hábitat natural del cultivo, lo que demuestra la alta variación que ha sufrido la composición arbórea natural del cacao, al estar asociado con especies frutales y forestales establecidas por los productores con intereses económicos por los cuantiosos usos: la alimentación, valor de la madera, medicina, entre otros (Somarriba et al., 2008).

En la estructura vertical del hábitat natural del cacao criollo, la altura que alcanzaban las especies asociadas es propia de las selvas tropicales, cuyas comunidades vegetales son las más exuberantes del país y la presencia de especies que integran el dosel con más de $30 \mathrm{~m}$ de altura y que conservan su follaje durante todo el año. Estas características de estratificación del hábitat natural del cacao nativo unido al nulo manejo del mismo, justifica la altura evaluada en los árboles de cacao. 
Los árboles del estrato superior pueden alcanzaron hasta $50 \mathrm{~m}$ de altura; varios de ellos son de importancia ecológico-social, como Ceiba pentandra (L.) Gaertn, Brosimum alicastrum Swartz, y Terminalia amazonia (J.F.Gmel.) Exell. (Román et al., 2009). Lo anterior hace posible que la morfología del cacao criollo fuera similar al de las especies arbóreas asociadas al mismo, ya que sus tallos crecen rectos y solo producen ramas cerca de la parte más alta. En este contexto, estudios realizados por Báez-Hernández et al. (2016) indicaron que "el tamaño y arquitectura de 19 especies de árboles reflejaron distintas estrategias de crecimiento dentro del gradiente vertical y horizontal del dosel y su coexistencia en la selva alta perennifolia de México". Esta sombra es causada por el dosel continuo del bosque maduro, también conocido como bosque primario. Por lo tanto, bajo estas condiciones se puede encontrar una vegetación muy densa, con grandes troncos de árboles intercalados con algunas plantas y arbustos en el sotobosque e incontables plantas pequeñas cerca del suelo (Butler, 2011).

En general, el manejo del agroecosistema cacaotero y sus constantes transformaciones en función del bienestar de los productores para obtener mayores bienes y servicios, ha repercutido en la separación del cultivo de sus condiciones originarias, aún cuando en la región que ocupa el Soconusco de Chiapas se encuentra la mayor parte de la vegetación natural como restos de selva. Ello se corrobora por la disimilitud demostrada en la composición arbórea entre ambos lugares, con tan solo dos especies compartidas.

Para entender los patrones ecológicos de la selva Lacandona a una escala regional relacionado con especies de interés genético como el cacao, es importante realizar estudios como estos a escalas locales en los que se describan y se cuantifiquen las características de los bosques primarios relacionados con las especies de interés (Stropp et al., 2009).

Los resultados expuestos son una contribución científica a considerar en el manejo y uso efectivo de las especies arbóreas asociadas al cacao que se cultiva en el Soconusco, Chiapas para lograr el diseño de sistemas agroforestales más eficientes y sustentables. Al mismo tiempo, los registros aquí anotados son evidencia de la importancia de los inventarios florísticos en ambas áreas estudiadas, cuyas colectas sistemáticas y permanentes de especies vegetales colaboran para mejorar el conocimiento botánico de la región.

El conocimiento obtenido a partir de los resultados de la presente investigación, permitirá ejecutar actividades de conservación in situ de los materiales de cacao nativo registrado, considerando su relación con la composición arbórea asociada, además de las estrategias de mejoramiento genético donde se logre el uso del germoplasma nativo único de México, que se caracteriza por ser un cacao fino de aroma. Estas estrategias revalorarán al recurso fitogenético del cacao y permitirán una conservación y uso sustentable.

\section{Conclusiones}

La abundancia, riqueza y densidad de especies arbóreas asociadas al cacao criollo es mayor que la registrada en los sistemas de producción. Ambas condiciones poseen solo dos especies compartidas que unido a las condiciones físicos-geográficas y tecnológicas, hacen posible la disimilitud encontrada entre las mismas.

Se registraron diferencias en la localización del cultivo del cacao dentro de la estructura vertical, pues a diferencia del cacao en los sistemas de producción que se encuentran en el estrato inferior, el cacao criollo se encontró en el estrato medio, posiblemente sea una estrategia natural de supervivencia por los factores ecológicos como la luz, para garantizar su conservación y preservación.

\section{Agradecimientos}

Al Fondo SADER-CONACYT-México por el financiamiento del proyecto 2017-02-291417, y a las facilidades de las autoridades de la Comisión Nacional de Áreas Naturales Protegidas (CONANP) y guarda parques del Parque Nacional Montes Azules. 


\section{Referencias}

Alvim, P. (1977). Cacao. In P. T. Alvim, \& T. T. Kozlowski (Eds.) Ecophysiology of Tropical Crops (pp. 279-313). Academic Press. https://doi.org/10.1016/C2013-0-07134-4

Avendaño-Arrazate, C. H., Martínez-Bolaños, M., \& Mendoza-López, A. (2018). The Cocoa (Theobroma cacao L.) native in México. Biodiversity International Journal, 2(6), 535-536. https://doi.org/10.15406/bij.2018.02.00109

Báez-Hernández, A., Herrera-Meza, G., Vázquez-Torres, M., Aquino-Bolaños, E. N., \& Martínez, A. J. (2016). Relaciones alométricas de 19 especies de árboles de la selva alta perennifolia. Botanical Sciences, 94(2), 209-220. https://doi. org/10.17129/botsci.252

Bonazzi, F., Hiroo, C., \& Rosa, R. H. (2014). Local tree preferences in the cacao-cabruca system in the southeast of Bahia, Brazil. Ambiente \& sociedade, 17(3), 55-78. http://www.researchgate.net/publication/269278476

Burnham, R. J. (2004). Alpha and beta diversity of lianas in Yasuni, Ecuador. Forest Ecology and Management, 190(1), $43-55$. https://doi.org/10.1016/j.foreco.2003.10.005

Butler, R. (2011). Rainforests (1 ${ }^{\text {st }}$ Ed.). Mongabay.

Comisión Nacional del Agua. (2015). Actualización de la disponibilidad media anual de agua en el acuífero Soconusco (0710) estado de Chiapas. http://www.gob.mx/cms/uploads/attachment/file/102911/DR_0710.pdf

Comisión Nacional para el Conocimiento y Uso de la Biodiversidad. (2018). Selvas húmedas. Biodiversidad mexicana. https:// www.biodiversidad.gob.mx/ecosistemas/selvaHumeda.html

Cordero, J., \& Boshier, D. H. (Eds.). (2003). Árboles de Centroamérica: Un manual para extensionistas (1 ${ }^{\text {a }}$ Ed.). Centro Agronómico Tropical de Investigación y Enseñanza. http://repositorio.bibliotecaorton.catie.ac.cr/handle/11554/9730

Curtis, J. T., \& McIntosh, R. P. (1951). An upland forest continuum in the prairie-forest border region of Wisconsin. Ecology, 32(3), 476-496. https://doi.org/10.2307/1931725

de-Oliveira, A. A., \& Nelson, B. W. (2001). Floristic relationships of terra firme forests in the Brazilian Amazon. Forest Ecology and Management, 146(1-3), 169-179. https://doi.org/10.1016/s0378-1127(00)00458-8

Durán-Fernández, A., Aguirre-Rivera, J. R., García-Pérez, J., Levy-Tacher, S., \& De Nova-Vásquez, J. A. (2016). Inventario florístico de la comunidad Lacandona de Nahá, Chiapas, México. Botanical Sciences, 94(1), 157-184. https://doi. org/10.17129/Botsci.248.

Escobar-Campos, M. C., \& Ochoa-Gaona, S. (2007). Estructura y composición florística de la vegetación del Parque Educativo Laguna Bélgica, Chiapas, México. Revista Mexicana de Biodiversidad, 78, 391-419. http://www.ib.unam.mx/m/ revista/pdfs/16-Escobar-122.pdf

Gayoso, J., \& Guerra, J. (2005). Contenido de carbono en la biomasa aérea de bosques nativos en Chile. Bosque, $26(2), 33-38$. https://dx.doi.org/10.4067/S0717-92002005000200005

García, E. (1973). Modificaciones del sistema de clasificación climática de Köppen para adaptarlo a las condiciones de la República Mexicana. Universidad Nacional Autónoma de México, \& Instituto de Geografía.

González, F. 2008. Ecofisiología del cacao. Blogspot. http://Diplomado2007unas.blogspot.com/2008/01/ecofisiología-delcacao.html 
Hernández Gómez, E., Hernández Morales, J., Avendaño Arrazate, C. H., López Guillen, G., Garrido Ramírez, E. R., Romero Nápoles, J., \& Nava Diáz, C. (2015). Factores socieconómicos y parasitológicos que limitan la producción del cacao en Chiapas, México. Revista Mexicana de Parasitología, 33(2), 232-246. http://www.scielo.org.mx/pdf/rmfi/ v33n2/2007-8080-rmfi-33-02-00232.pdf

Levy-Tacher, S. I., Aguirre Rivera, R. J., Martínez Romero, R. M., \& Durán Fernández, F. A. (2002). Caracterización del uso tradicional de la flora espontánea en la comunidad Lacandona de Lacanha, Chiapas, México. Interciencia, 27(10), 512-520. http://www.scielo.org.ve/scielo.php?script=sci_arttext\&pid=S0378-18442002001000002

Levy-Tacher, S. I., Aguirre-Rivera, R. J., García-Pérez, J. D., \& Martínez-Romero, M. M. (2006). Aspectos florísticos de Lacanhá Chansayab, Selva Lacandona, Chiapas. Acta Botánica Mexicana, 77, 69-98. http://www.redalyc.org/articulo. oa? id=57407705

López-Baez, O., Ramírez-Gonzaléz, S., Espinosa-Zaragoza, S., Villarreal-Fuentes, J., \& Wong-Villarreal, A. (2015). Diversidad vegetal y sustentabilidad del sistema agroforestal de cacao en la región de la selva de Chiapas, México. Revista Iberoamericana de Ciencias, 2, 55-63.

Nebel, G., Kvist, L. P., Vanclay, J. K., \& Vidaurre, H. (2001a). Forest dynamics in flood plain forests in the Peruvian Amazon: effects of disturbance and implications for management. Forest Ecology and Management, 150(1-2), 79-92. https:// doi.org/10.1016/s0378-1127(00)00682-4

Nebel, G., Kvist, L. P., Vanclay, J. K., Christensen, H., Freitas, L., \& Ruı́z, J. (2001b). Structure and floristic composition of flood plain forests in the Peruvian Amazon. Forest Ecology and Management, 150(1-2), 27-57. https://doi. org/10.1016/s0378-1127(00)00680-0

Ochoa-Gaona, S., Hernández-Vázquez, F., De Jong, B. H. J., \& Gurrí-García, F. D. (2007). Pérdida de diversidad florística ante un gradiente de intensificación del sistema agrícola de roza-tumba-quema: un estudio de caso en la Selva Lacandona, Chiapas, México. Boletín de la Sociedad Botánica de México, 81, 65-80. https://doi-org/10.17129/botsci.1766

Parolin, P., De Simone, O., Haase, K., Waldhoff, D., Rottenberger, S., Kuhn, U., Kesselmeier, J., Kleiss, B., Schmidt, K., Piedade, MT F., \& Junk, W. J. (2004). Central Amazonian floodplain forests: Tree adaptations in a pulsing system. The Botanical Review, 70(3), 357-380. https://doi.org/10.1663/0006-8101(2004)070[0357:CAFFTA]2.0.CO;2

Pennington, T. D., \& Sarukhán, J. (2005). Árboles tropicales de México: manual para la identificación de las principales especies. Universidad Nacional Autónoma de México.

Pitman, N. CA., Terborgh, J. W., Silman, M. R., Nuñez, P., Neill, D, Cerón, C., Waler, P., \& Aulestia, M. (2002). A comparison of the tree species diversity in two upper Amazonian forests. Ecology, 83(11), 3210-3224. https://doi.org/10.1890/00129658(2002)083[3210:ACOTSD]2.0.CO;2

Polo-Urrea, C. S. (2008). Índices más comunes en biología. Segunda parte, similaridad y riqueza beta y gamma. Revista de la Facultad de Ciencias Básicas, 4(1), 135-142. http://www.dialnet.unirioja.es/servlet/articulo?codigo=3050699

Poulsen, A. D., Tuomisto, H., \& Balslev, H. (2006). Edaphic and floristic variation within a 1-ha plot of lowland Amazonian rain forest. Biotropica, 38(4), 468-478. https://doi.org/10.1111/j.1744-7429.2006.00168.x

Ramírez-Meneses, A., García-López, E., Obrador-Olán, J. J., Ruiz-Rosado, O., \& Camacho-Chiu, W. (2013). Diversidad florística en plantaciones agroforestales de cacao en Cárdenas, Tabasco, México. Universidad y Ciencia, 29(3), 215230. http://www.scielo.org.mx/pdf/uc/v29n3/v29n3a1.pdf 
Rice, R., \& Greenberg, R. (2000). Cultivo de cacao y conservación de la diversidad biológica. Ambio, 29(3), 167-173. https:// doi.org/10.1639/0044-7447(2000)029[0167: CCATCO]2.0.CO; 2.

Roa-Romero, H. A., Salgado-Mora, M. G., \& Álvarez-Herrera, J. (2009). Análisis de la estructura arbórea del sistema agroforestal de cacao (Theobroma cacao L.) en el Soconusco, Chiapas, México. Acta Biológica Colombiana, 14(3), 97-110. http://revistas.unal.edu.co/index.php/actabiol/article/view/12599/13199

Román, F. J., Levy, S. I, Aguirre, J. R., \& Sánchez, A. (2009). Árboles de la Selva Lacandona útiles para la restauración ecológica. Colegio de la Frontera Sur. http://centro.paot.org.mx/documentos/conafor/Arboles-Selva-Lacandona.pdf

Romaní-Cortes, J. (2009). De necedades y necesidades. Anatomía de un conflicto agrario-ambiental en la zona norte de la Lacandona (Serie Acciones No 4). Comisión Nacional para el Conocimiento y Uso de la Biodiversidad, \& Corredor Biológico Mesoamericano México.

Salgado-Mora, M. G., Ibarra Núñez, G., Macías-Sámano, J. E., \& López-Báenz, O. (2007). Diversidad arbórea en cacaotales del Soconusco, Chiapas, México. Interciencia, 32(11), 763-768. http://www.redalyc.org/articulo.oa?id=33901107

Sánchez-Gutiérrez, F., Pérez-Flores, J., Obrador Olan, J. J., Sol Sánchez, A., \& Ruiz-Rosado, O. (2016). Árboles maderables en el sistema agroforestal de cacao en Cárdenas, Tabasco, México. Revista Mexicana de Ciencias Agrícolas, 14(Esp.), 2711-2723. http://www.scielo.org.mx/pdf/remexca/v7nspe14/2007-0934-remexca-7-spe14-2711-en.pdf

Schupp, E. W., Jordano, P., \& Gómez, J. M. (2010). Seed dispersal effectiveness revisited a conceptual review. New Phytologist, 188(2), 333-353. https://doi.org/10.1111/j.1469-8137.2010.03402.x

Servicio de Información Agroalimentaria y Pesquera. (2018). Intención de cosecha de cultivos perennes por estado en México 2018. https://infosiap.siap.gob.mx/opt/agricultura/intension/Intencion_cosechaPerenne_estado2018.pdf

Silva, C., Orozco, L., Rayment, M., \& E. Somarriba. E. (2013). Conocimiento local sobre los atributos deseables de los árboles y el manejo del dosel de sombra en los cacaotales de Waslala, Nicaragua. Agroforestería en las Américas, 49, 51-60.

Somarriba, E. (2002). Estimación visual de la sombra en cacaotales y cafetales. Agroforestería en las Américas, 9, 84-94.

Somarriba, E. (2004). ¿Cómo evaluar y mejorar el dosel de sombra? Agroforestería en las Américas, 41-42, 122-130. http:// repositorio.bibliotecaorton.catie.ac.cr/handle/11554/6941

Somarriba, E. J., Andrade, H. J., Segura, M., \& Villalobos, M. (2008). ¿Cómo fijar carbono atmosférico, certificarlo y venderlo para complementarlos ingresos de productores indígenas de Costa Rica? Revista Agroforestería en las Américas, 46, 81-88. http://repositorio.bibliotecaorton.catie.ac.cr/handle/11554/10234

Somarriba, E., Beer, J., \& Muschler, R. (2000). Problemas y soluciones metodológicas en la investigación agroforestal con café y cacao en CATIE. Agroforestería en las Américas, 7(25), 27-32. https://www.researchgate.net/publication/324174451_ Problemas_y_soluciones_metodologicas_en_la_investigacion_agroforestal_con_cafe_y_cacao_en_CATIE

Somarriba, E., Harvey, C., Samper, M., Anthony, F., Gonzalez, J., Stracer, C., \& Rice, R. (2004). Conservation of biodiversity in neotropical coffee (Coffea arabica) plantations. In G. Schroth, G. A. B. Da Fonseca, C. A. Harvey, C. Gascon, H. L. Vasconcelos, \& A. M. N. Izac (Eds.), Agroforestry and boidiversity conservation in tropical landscapes (pp. 198226). Island Press.

Stiling, P. D. (1999). Ecology: theories and applications. Prentice Hall.

Stropp, J., Ter Steege, H., \& Malhi, Y. (2009). Disentangling regional and local tree diversity in the Amazon. Ecography, 32(1), 46-54. https://doi.org/10.1111/j.1600-0587.2009.05811.x 
Suárez-Venero, G. M., Avendaño-Arrazate, C. H, Ruiz-Cruz, P. A., \& Estrada-de-los-Santos, P. (2018). Estructura e impacto de la diversidad taxonómica en cacao del Soconusco, Chiapas, México. Agronomía Mesoamericana, 30(2), 353-365. https://doi.org/10.15517/amv30i2.34032

Wittmann, F., Schongart, J., Montero, J. C., Motzer, T., Junk, W. J., Piedade, M. T. F., Queiros, H. L., \& Worbes, M. (2006). Composición de especies de árboles y gradientes de diversidad en bosques de aguas bravas en la Cuenca del Amazonas. Revista de Biogeografía, 33(8), 1334-1347. https://doi.org/10.1111/j.1365-2699.2006.01495.x 